\title{
Longitudinal Evaluation of Health Plan Cost per Venous Thromboembolism or Bleed Event in Patients With a Prior Venous Thromboembolism Event During Hospitalization
}

\author{
MICHAEL F. BULLANO, PharmD; VINCENT WILLEY, PharmD; OLE HAUCH, MD; GAIL WYGANT, MS, RN;
} ALEX C. SPYROPOULOS, MD; and LAUREN HOFFMAN, PharmD

\begin{abstract}
OBJECTIVE: To measure the per-event health plan costs for acute and follow-up treatment not directed by a clinical study protocol in a group of commercially insured patients in 2 managed care organizations following an incident hospitalization that included a diagnosis for a venous thromboembolism (VTE) event.

METHODS: A cohort of patients with an incident in-hospital VTE event, consisting of deep vein thrombosis (DVT), or pulmonary embolism (PE), or both DVT + PE, was retrospectively identified from the administrative claims databases of 2 large U.S. health care plans. Inclusion criteria were (a) an inpatient VTE event between January 1, 1998, and December 31, 2000, (b) no VTE diagnosis or anticoagulation therapy 3 months prior to the incident VTE in-hospital event, (c) at least 1 anticoagulation pharmacy fill following the incident hospital VTE, and (d) continuous health plan enrollment 3 months prior to and 6 months following the incident hospital VTE event. Total costs were reported on a per-event basis and consisted of the aggregated amount paid by the health plan to the provider after subtraction of member cost-share. Costs were collected separately, first for the incident VTE event for all patients identified and second for patients who had at least 1 of the following events in the follow-up period: bleed requiring or not requiring hospitalization, a recurrent VTE event requiring hospitalization, or a recurrent VTE and bleed (VTE + bleed) event requiring hospitalization. Costs were compared between incident diagnosis groups using multivariate generalized linear model techniques.
\end{abstract}

RESULTS: A total of 2,147 patients (DVT =1,499 [69.8\%], PE $=373$ [17.4\%], DVT + PE= $275[12.8 \%]$ ) were identified (mean age $=61.6 \pm$ standard deviation $[S D] 16$ years; $46.3 \%$ male) and were followed for an average of 21.3 (median, 19.2) months. Disease severity was high in these patients, including $59.2 \%$ with a history of or active malignancy. The prevalence of VTE was 2.04 per 100,000 study-eligible health plan members. For the incident VTE events, average costs were $\$ 7,712 \pm \$ 18,339$ (median, $\$ 3,131$ ) per incident DVT event; $\$ 9,566 \pm \$ 13,512$ (median, $\$ 6,424$ ) per PE incident event; and $\$ 12,200 \pm \$ 24,038$ (median, $\$ 6,678$ ) per incident DVT+PE event. Warfarin treatment following the incident VTE event was administered to $97.3 \%$ of patients for an average of 6.7 (median, 5.0) months at an average cost of $\$ 19.40$ per patient per month. During the average period of 21.3 months, 534 patients (24.9\%) experienced an average of 1.24 bleed or recurrent VTE events per patient that required hospitalization at a mean cost of $\$ 14,975$ per event or $\$ 2,101$ per patient per year. For patients with a bleed in the follow-up period that required hospitalization, average costs were $\$ 12,326 \pm \$ 24,448$ (median, $\$ 5,736$ ) per recurrent VTE; $\$ 15,339 \pm \$ 52,029$ (median, $\$ 4,999$ ) per bleed; or $\$ 24,085 \pm \$ 65,411$ (median, $\$ 10,185$ ) per recurrent VTE + bleed event. During the follow-up period, a total of 612 patients (28.5\%) experienced 1,489 recurrent bleed events that did not require hospitalization, at an average cost of \$239 $\pm \$ 386$ (median, \$95) per event. There were no significant differences in mean total costs for all pair-wise comparisons between the 3 incident diagnosis groups.

CONCLUSIONS: Of patients who experienced a VTE event during the incident hospital stay for any diagnosis, 1 in 4 experienced an average of 1.24 bleed or recurrent VTE events that required hospitalization in the 21 months of follow-up and incurred an average health plan cost of $\$ 14,957$ per event. These data may be of interest to managed care decision makers when evaluating the cost impact of new therapies or providing more comprehensive anticoagulation management services for existing therapies.

KEYWORDS: Anticoagulants, Thrombosis, Thromboembolism, Pulmonary embolism, Deep vein thrombosis, Outcomes research, Retrospective study, Managed care, Cost

J Manag Care Pharm. 2005;11(8):663-73

Note: An editorial on the subject of this article appears on pages 704-08 of this issue.
$\mathrm{P}$ ulmonary embolism (PE) and deep vein thrombosis (DVT), collectively known as venous thromboembolism (VTE), are significant causes of disability and death in the United States, resulting in approximately 300,000 hospitalizations and at least 50,000 deaths per year. ${ }^{1-3}$ It is estimated that direct medical care alone costs the country's health care systems at least $\$ 600$ million annually. ${ }^{4}$ In addition, among patients adequately treated for VTE, thromboembolism may recur in $5 \%$ at 3 months and up to $30 \%$ at 8 years. ${ }^{5-7}$ It has been suggested that, in DVT patients alone, nearly $\$ 500,000$ in health care costs could be prevented per 100 patients per year if patients were properly screened and treated, a process that may prevent high recurrent event costs.

The primary short-term goals of DVT therapy are to avoid the advancement of existing thrombi, the development of fatal PEs, and the early recurrence of thrombotic disease. The longterm treatment objective is to prevent delayed recurrence of DVT and PE (PE events as well as potential sequelae such as postphlebitic syndrome and pulmonary hypertension). ${ }^{9}$ Effective regimens for acute treatment include unfractionated heparin (UFH), low-molecular-weight heparin (LMWH), or adjusted-dose subcutaneous heparin. For initial VTE, acute therapy should be followed by warfarin therapy for at least 3 months if warfarin therapy is not contraindicated. Growing understanding of treatment for VTE has led to guidelines-an initial set in 1998, updated in 2001. ${ }^{10,11}$ The 1998 and 2001 guidelines were similar with respect to the intensity of oral pharmacotherapy used in prophylaxis after a VTE event.

\section{Authors}

MICHAEL F. BULLANO, PharmD, is director, health outcomes research, and VINCENT WILLEY, PharmD, is vice president, research, HealthCore, Wilmington, Delaware; OLE HAUCH, MD, and GAIL WYGANT, MS, RN, are directors, health economics and outcomes research, AstraZeneca LP, Wilmington, Delaware; ALEX C. SPYROPOULOS, MD, is director, antithrombotic services, Lovelace Medical Center, Albuquerque, New Mexico; LAUREN HOFFMAN, PharmD, is director, clinical pharmacy services, BlueCross and BlueShield of Florida, Inc., Jacksonville.

AUTHOR CORRESPONDENCE: Michael F. Bullano, PharmD, HealthCore, Inc 800 Delaware Ave., Wilmington, DE 19801. Tel: (302) 230-2123; Fax: (302) 230-2020; E-mail: mbullano@healthcore.com

Copyright $@ 2005$, Academy of Managed Care Pharmacy. All rights reserved 


\section{Longitudinal Evaluation of Health Plan Cost per Venous Thromboembolism or Bleed Event in Patients With a Prior Venous Thromboembolism Event During Hospitalization}

However, the 2001 guidelines added the confirmation of LMWH preparations as replacements for UFH in venous thromboembolism. ${ }^{11}$

As health care systems continue to strive to reduce the rate of increase in overall health care expenditures, particularly the cost of hospital care, costs associated with both initial VTE care and follow-up care with related sequelae are becoming increasingly important to formulary and health policy decision makers. ${ }^{12}$ Emphasis is being placed on safe and effective, yet cost-efficient treatments that represent the interests of both the health care system and the patient. ${ }^{13}$ A number of cost-effectiveness analyses have outlined VTE inpatient and outpatient management costs, ${ }^{8,14,15}$ and others have outlined the cost-effectiveness of LMWHs, warfarin therapy, and unfractionated heparins ${ }^{16-19}$; however, little research exists evaluating the period following an incident VTE for recurrent VTE events, bleed events, or therapy monitoring in a real-world setting. This study was designed to follow a group of commercially insured patients in 2 managed care organizations (MCOs) following an incident VTE, measuring the costs for acute and follow-up treatment undirected by protocol.

\section{Methods}

\section{Study Design and Location}

We conducted a retrospective, observational cohort study covering the period from October 1, 1997, through September 30, 2001. Electronic medical and pharmacy administrative claims from 2 health plans located in the southeast and western United States, with approximately 3.5 million and 7.5 million covered members, respectively, were extracted and used in this study. The health plans were similar in overall benefit design, types of health insurance products offered, and administrative claim data elements available for analysis. Both health plans had an independent practice association structure that largely comprised a health maintenance organization (HMO) and, to a lesser extent, preferred provider organization (PPO) members and point-of-service members. Some providers (hospital and physicians) operated under a prearranged or capitated reimbursement structure. In addition, both plans offered a Medicare + Choice (now Medicare Advantage) benefit (for those aged $\geq 65$ years). Neither health plan directly sponsored any specific programs or interventions targeting anti-coagulation therapy during the time period of this study.

\section{Study Population}

This study included all members of the 2 MCOs for whom administrative claims data included a hospital claim with a primary or secondary diagnosis for either a DVT and/or PE during a hospitalization for any cause that occurred between January 1, 1998, and December 31, 2000. We designated the incident (i.e., first) claim for a member during the study period as the index diagnosis. Diagnosis of inpatient DVT or PE was determined by the presence of (a) International Classification of Diseases, Ninth Revision (ICD-9) codes of 451.1x for DVT and 415.1x for $\mathrm{PE}$, in position 1 (primary) or 2 (secondary); (b) universal billing (1992 revision UB-92) codes associated with an inpatient hospitalization (100, 101, or 110); and (c) at least 1 pharmacy claim for an anticoagulation medication (Generic Product Identifier [GPI] $=83$ ) $^{20}$ in the 30-day period following the index diagnosis.

Patients were excluded from the study if they had been diagnosed with a VTE or had used any anticoagulation therapy in the 3 months preceding the index diagnosis or if they did not have continuous MCO eligibility for at least 3 months prior to and 6 months following the index diagnosis.

For purposes of analysis, patients were assigned to 1 of 3 groups based on their index diagnosis. The DVT group included patients who had an index diagnosis containing only ICD-9 codes (451.1x) for DVT (but not for PE), the PE group included all patients with an index diagnosis containing only ICD-9 codes (415.1x) for PE (but not for DVT), and the DVT + PE group included patients with ICD-9 codes for both DVT and PE (415.1x, 451.1x). In addition, patient identifiers were masked to protect the identity of individual health information.

We followed patients longitudinally from the index diagnosis to the end of benefit eligibility or to the end of the study period (September 30, 2001), whichever occurred first. We collected baseline data from the index date back to the patient's beginning of eligibility or the beginning of the study period (October 1 , 1997), whichever occurred first. To further describe the case mix of our population, surrogate measures of disease severity were measured during the preindex period. Collected measures included the number of distinct medications filled (i.e., distinct by chemical entity) and the total amount paid for all pharmacy and medical claims (regardless of diagnosis) by the health plan. These surrogate measures represent a measure of the burden of comorbid diseases, and their utility has been explained and compared previously. ${ }^{21}$ In addition, we captured the prevalence of known independent risk factors for recurrent thromboembolism $^{22}$ by reviewing all medical and pharmacy encounters over the observation period.

Following the index diagnosis, we documented all bleed and recurrent VTE events based on the appearance of ICD-9 codes in the MCO claims data. We used the "place of service" field to determine whether the medical service occurred in the hospital, with or without subsequent skilled nursing care but not including direct admission to a skilled nursing facility (SNF), or in an outpatient setting. Postindex events were categorized as (1) recurrent VTE events requiring hospitalization (no bleed codes observed), (2) bleed events requiring hospitalization (no VTE codes observed), (3) VTE and bleed (VTE + bleed) events requiring hospitalization (codes for both VTE and bleed observed), and (4) bleed events not requiring hospitalization (code for bleed observed; codes related to VTE may or may not 


\section{Longitudinal Evaluation of Health Plan Cost per Venous Thromboembolism or Bleed Event in Patients With a Prior Venous Thromboembolism Event During Hospitalization}

be observed). For each event of interest, the corresponding series of claim records were assembled and the respective amount paid per event was aggregated using the "amount paid" (by the health plan to the provider after subtraction of the member cost-share) field within the administrative claims database (i.e., the patients' coinsurance, copayments, and deductibles were not included in any of the cost calculations).

\section{Cost and Clinical Outcomes}

The primary cost outcome was the aggregated cost per event from the "amount paid" field in those patients requiring hospitalization for such events. Secondary cost outcomes included (1) the cost of the index VTE event, (2) outpatient anticoagulation therapy and related monitoring costs, and (3) the costs of treating bleed events not requiring hospitalization. To be counted, a claim must have had at least 1 ICD-9 code for a bleed (Table 1). Medical claims that had dates of service that were separated by more than 1 day were counted as separate encounters. In addition, the patient must have been receiving anticoagulation therapy at the time of the bleed occurrence.

To examine determinants of the total cost of care for postindex events requiring a hospital visit, aggregated costs were broken into 5 resource centers based on the individual UB-92 code assigned to individual paid amounts. For hospital admissions that were paid on a prospective basis (i.e., per diem), costs for the individual resource centers were calculated by multiplying the percentage that the resource center charge contributed to the total hospital charge by the total amount paid. For example, if the hospital charged $\$ 6,000$ and was paid $\$ 4,000$ for the entire admission on a prospective basis, and the claim line for room and board showed a charge of $\$ 3,000$, room and board would have contributed $50 \%$ of the total charge, and the cost for room and board would have been calculated to be $\$ 2,000$.

Emergency department included all emergent and urgent care services (UB-92 range: 450-459); room and board included all costs related to critical care units, wards, nursing, monitoring, and supply costs (UB-92 range: 100-249, 279-299, 620-624, 700-770); diagnostic and laboratory included all laboratory and diagnostic tests, such as radiographic tests, Doppler ultrasound, computed tomography, resonance-based diagnostic tests, and peripheral vascular laboratory costs (UB-92 range: 300-359, $402-409,610-619,921)$; and pharmacy (Rx) included all medication costs, specific medications not specified, and intravenous supplies and administration (UB-92 range: 250-269). All paid amounts that contained UB-92 codes that did not fit any other category, including operating room, anesthesia, dialysis, oxygen, and professional fees, were designated as "other."

Outpatient international normalized ratio (INR) tests were identified in the administrative medical claims data utilizing the Current Procedural Terminology (CPT) code of 85610, and the

\begin{tabular}{r|l}
\hline \multicolumn{1}{c}{ TAB LE 1 ICD-9 Codes for Bleed Events } \\
\hline ICD-9 & Bleed Description \\
\hline 287.9 & Unspecified hemorrhagic conditions \\
360.43 & Hemophthalmos, except current injury \\
362.81 & Retinal hemorrhage \\
372.72 & Conjunctival hemorrhage \\
374.81 & Hemorrhage of eyelid \\
376.32 & Orbital hemorrhage \\
430 & Subarachnoid hemorrhage \\
431 & Intracerebral hemorrhage \\
432 & Other and unspecified intracranial hemorrhage \\
448.9 & Other and unspecified capillary diseases \\
459.0 & Unspecified hemorrhage \\
523.8 & Other specified periodontal diseases \\
530.82 & Esophageal hemorrhage \\
531.0 & Acute gastric ulcer with hemorrhage \\
531.4 & Chronic or unspecified gastric ulcer with hemorrhage \\
532.0 & Acute duodenal ulcer with hemorrhage \\
532.4 & Chronic or unspecified duodenal ulcer with hemorrhage \\
533.0 & Acute peptic ulcer, unspecified site, with hemorrhage \\
533.4 & Chronic or unspecified peptic ulcer, unspecified site, with \\
& hemorrhage \\
562.02 & Diverticulosis of small intestine with hemorrhage \\
562.03 & Diverticulitis of small intestine with hemorrhage \\
562.12 & Diverticulosis of colon with hemorrhage \\
562.13 & Diverticulitis of colon with hemorrhage \\
569.3 & Hemorrhage of rectum and anus \\
596.7 & Gastrointestinal hemorrhage \\
599.89 & Hemorrhage into bladder wall \\
782.7 & Other specified disorders of urinary tract \\
784.7 & Epontaneous ecchymoses \\
784.8 & Hemorrasis \\
786.3 & Hemoptysis \\
\hline & Subarachnoid, subdural, and extradural hemorrhage, following \\
\hline injury & Secondary and recurrent hemorrhage as an early complication \\
& of trauma \\
\hline
\end{tabular}

ICD-9=International Classification of Diseases, Ninth Revision.

"amount paid" field was captured to represent the cost. All anticoagulant medication costs (warfarin, UFH, and LMWH) were identified from the pharmacy claims database utilizing the appropriate GPIs (starting with 83), and costs were assigned using the "amount paid" field. In these particular health plans, anticoagulant utilization and costs could not be ascertained from the medical administrative claims database for the inpatient setting because all pharmacy charges for a hospital admission were aggregated on a single claim for that hospitalization. The pharmacy administrative claims database was utilized solely to determine the anticoagulant costs for outpatient care because both plans covered claims for anticoagulants under the pharmacy benefit. 


\section{TABLE 2 Sample Selection}

\begin{tabular}{|c|c|c|}
\hline Criteria & $\begin{array}{l}\text { Number (\%) } \\
\text { of Patients } \\
\text { Remaining }\end{array}$ & $\begin{array}{l}\text { Number } \\
\text { of Patients } \\
\text { Dropped }\end{array}$ \\
\hline $\begin{array}{l}\text { Patients must have had at least } 1 \text { VTE } \\
\text { diagnosis between January 1, 1998, and } \\
\text { December 31, } 2000\end{array}$ & $26,103(100)$ & - \\
\hline $\begin{array}{l}\text { Patients must not have had a VTE diagnosis } \\
\text { in the } 3 \text { months prior to their index date }\end{array}$ & $25,784(98.8)$ & $319(1.2)$ \\
\hline $\begin{array}{l}\text { Patients must not have been receiving an } \\
\text { anticoagulation medication in the } 3 \text { months } \\
\text { prior to their index hospital event }\end{array}$ & $23,564(90.3)$ & $2,220(8.5)$ \\
\hline $\begin{array}{l}\text { Patients must have been continuously enrolled } \\
\text { in a health plan (for a period of } 3 \text { months prior } \\
\text { to and } 6 \text { months following their VTE index date) }\end{array}$ & $15,850(60.7)$ & $7,714(29.6)$ \\
\hline $\begin{array}{l}\text { Patients must have been exposed to at least } 1 \text { fill } \\
\text { of a VTE anticoagulation medication following } \\
\text { their index date }\end{array}$ & $2,147(8.2)$ & $13,703(52.5)$ \\
\hline
\end{tabular}

\section{Anticoagulation Therapy and Monitoring}

Duration of anticoagulation therapy was captured for patients prescribed warfarin and/or outpatient LMWH therapy. Because the daily dose of warfarin therapy is changed frequently between individual prescription refills, conventional approaches of determining duration of therapy that incorporate only days supply and fill dates may not be accurate. Therefore, we employed the following logic to determine duration of therapy. The first warfarin prescription date was identified in the follow-up period. From this date, all pharmacy and laboratory claims were reviewed in the subsequent 42 days ( 6 weeks) for the presence of a warfarin prescription or a laboratory claim for a prothrombin time/international normalized ratio (PT/INR). We selected 6 weeks a priori based on a prior study evaluating the validity of this approach in determining continuous warfarin therapy. In that study, the concordance between the 42-day algorithm and actual continuous warfarin therapy was very high $(\kappa=0.84){ }^{23}$ If one of these conditions was met, the same process continued from the date of the new warfarin prescription or the PT/INR. This logic was applied to a patient's records until both conditions were not met; the date of last warfarin fill or PT/INR was recorded. The duration of warfarin therapy was calculated from the date difference between the first and last warfarin prescriptions plus days supply of the last prescription or the date of the last PT/INR test, whichever was greater. Respective costs representing paid amounts to pharmacy providers were captured and aggregated.

Outpatient monitoring consisted of capturing the utilization and respective costs of PT/INR tests. Patient records that exhibited warfarin use were scanned for a CPT code of 85610 to denote the presence of a PT/INR test. Costs for antifactor Xa levels were not captured because of the lack of a distinct CPT code during the period of extracted data.

\section{Statistical Analyses}

Descriptive statistics included mean ( \pm standard deviation [SD]) and median values for continuous data and relative frequencies for categorical data. Continuous variables were compared between index groups with analysis of variance (ANOVA) with the Scheffe test for multiple comparisons. This post hoc test was chosen because it is more stringent (i.e., less likely to commit an $\alpha$ error) as compared with other tests. ${ }^{24}$ Due to the nonnormal distribution of the data, we also examined results from Kruskal-Wallis statistical analyses. Because our data set was large, results from the 2 tests were equivalent, and we have elected to provide the more commonly used ANOVA. Categorical variables were compared using Pearson $\chi^{2}$ tests. Rates of INR use were created by dividing the total number of PT/INR tests by each individual's time of observation following the index diagnosis. Rates were compared by calculating 95\% confidence intervals using the Poisson Exact methodology. ${ }^{25}$ For these procedures, statistical significance was defined a priori at an $\alpha$ of $<0.05$.

To determine if costs for postindex events were different between index diagnoses, multivariate generalized linear model techniques were used. Because prior research supports the observation that cost data appear to follow a $\gamma$ distribution, we modeled cost outcomes using a general linear model (GLM) with a log link and $\gamma$ distribution. ${ }^{26}$ The 4 cost outcomes modeled were (1) recurrent VTE alone requiring hospitalization, (2) bleed events alone requiring hospitalization, (3) recurrent VTE + bleed events requiring hospitalization, and (4) bleed events not requiring hospitalization. Model covariates included index diagnosis, age, gender, presence of risk factors for thrombosis observed in any period, history of bleed in the 3 months prior to the index VTE, and number of distinct medications at baseline. Postindex observation time was not introduced into the model because costs were analyzed on a per-event basis. We fit general linear models with all variables with Wald $P$ values $<0.15$ (after eliminating collinear variables). For the final estimating model, we retained the set of variables all having Wald $P$ values $<0.15$ and the largest negative Bayesian Information Criteria score for the model. ${ }^{27}$ All statistical analyses were performed using STATA $7.0 .^{28}$

\section{Results}

\section{Population}

Table 2 shows the sample selection. A total of 2,147 patients were included in our study: 887 and 1,260 from the southeastern and western health plans, respectively, representing an incidence of approximately 57 per 100,000 study-eligible members in the southeastern health plan and 41.6 per 100,000 studyeligible members in the western health plan. Baseline characteristics appear in Table 3. Of the 2,147 patients, 1,499 (69.8\%) had an index diagnosis of DVT, 373 (17.4\%) had an index diagnosis of $\mathrm{PE}$, and 275 (12.8\%) patients had an index 


\section{Longitudinal Evaluation of Health Plan Cost per Venous Thromboembolism or Bleed Event in Patients With a Prior Venous Thromboembolism Event During Hospitalization}

\section{TABLE 3 Cohort Characteristics Stratified by Index Diagnosis $(N=2,147)$}

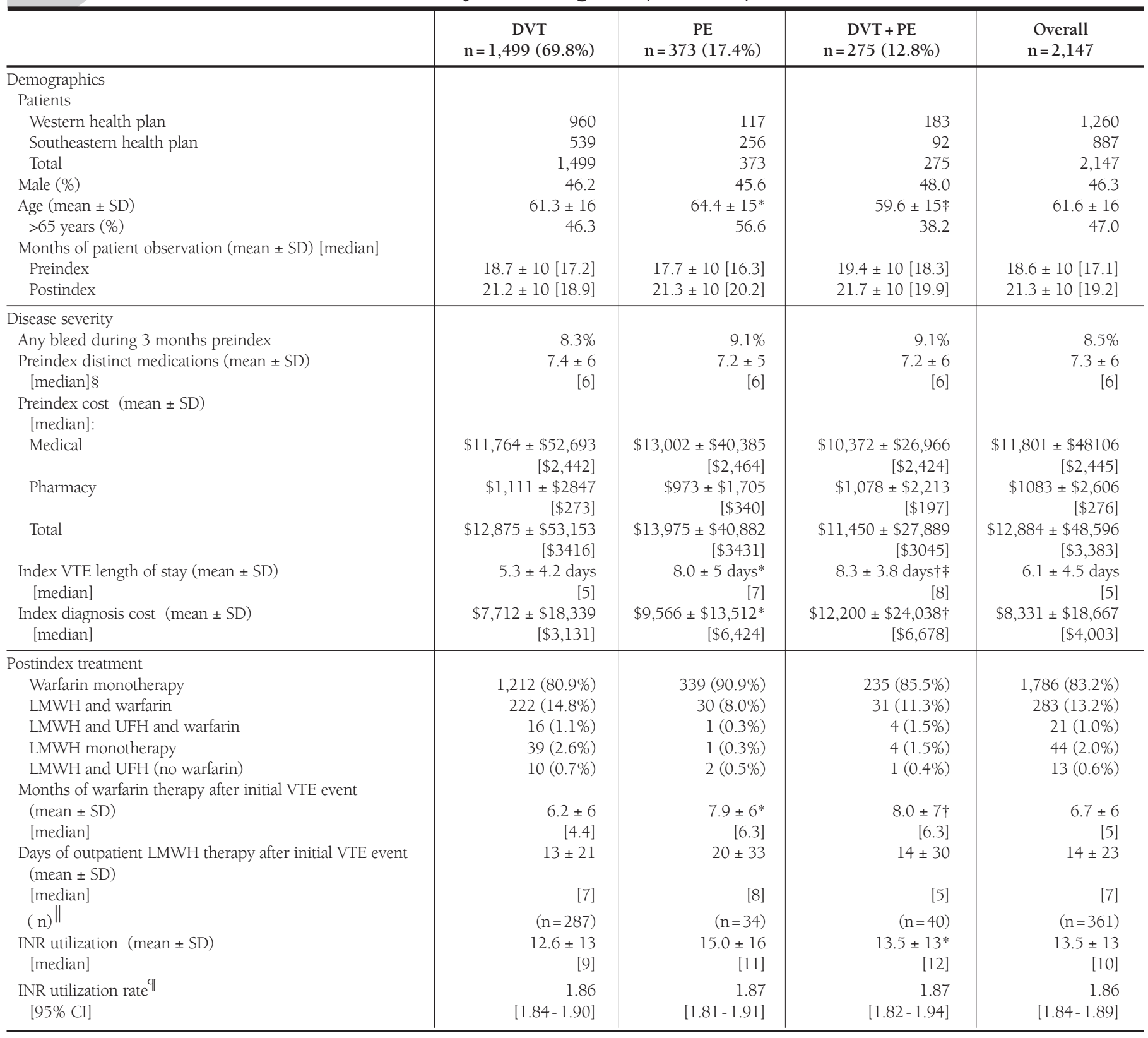

* Significance compared with DVT group at $P<0.05$ level (PE vs. DVT).

+ Significance compared with DVT group at $P<0.05$ level (DVT + PE vs. DVT)

$¥$ Significance compared with $P E$ group at $P<0.05$ level (DVT $+P E$ vs. $P E)$.

$\S$ Distinct chemical entities.

$\|$ Calculated for patients who received an outpatient course of LMWH.

II INR per patient per month.

$C I=$ confidence interval; $D V T=$ deep vein thrombosis; $I N R=$ international normalized ratio; $L M W H=l o w$-molecular-weight heparin; $P E=$ pulmonary embolism;

UFH=unfractionated heparin; VTE=venous thromboembolism.

Data are presented as mean \pm standard deviation [median] or column percentage. All costs were collected from the "amount paid" fields from the administrative claims databases. Preindex costs include amount paid for all claims regardless of diagnosis (i.e., all-cause). Index event costs are amount paid for inpatient claims with the specific index diagnosis. 


\section{Longitudinal Evaluation of Health Plan Cost per Venous Thromboembolism or Bleed Event}

in Patients With a Prior Venous Thromboembolism Event During Hospitalization

\begin{tabular}{l|c|c|c|c}
\hline TAB LE 4 Risk Factors Stratified by Index \\
VTE Diagnosis (N= 2, 147) \\
\hline & DVT & PE & DVT + PE & Overall \\
& $(\%)$ & $(\%)$ & $(\%)$ & $(\%)$ \\
Risk Factor & $\mathbf{n = 1 , 4 9 9}$ & $\mathbf{n = 3 7 3}$ & $\mathbf{n}=\mathbf{2 7 5}$ & $\mathbf{n = 2 , 1 4 7}$ \\
\hline History of or active malignancy & 59.8 & 56.8 & 59.3 & 59.2 \\
Other cardiac diseases & 53.4 & 59.8 & 58.9 & $55.2^{*}$ \\
Coagulation defects & 27.0 & 30.6 & 34.9 & $28.6^{*}$ \\
Trauma & 24.0 & 26.3 & 24.7 & 24.5 \\
Atrial fibrillation & 21.0 & 28.7 & 25.5 & $22.9 *$ \\
Congestive heart failure & 20.0 & 30.8 & 23.3 & $22.3 *$ \\
Contraception or estrogen & 22.2 & 20.9 & 21.1 & 21.8 \\
therapy use & & & & \\
Ischemic stroke & 20.4 & 24.1 & 17.1 & 20.6 \\
Inflammatory bowel disease & 12.8 & 10.2 & 10.2 & 12.0 \\
Varicose veins & 12.3 & 9.65 & 8.73 & 11.4 \\
Myocardial infarction & 9.47 & 14.5 & 9.09 & $10.3 *$ \\
Surgery & 7.27 & 5.36 & 5.82 & 6.75 \\
Hyperviscosity syndromes & 2.47 & 2.95 & 3.27 & 2.65 \\
Pregnancy & 1.47 & 1.07 & 1.09 & 1.35 \\
\hline
\end{tabular}

Risk factors were assessed over entire observation period (both preindex and postindex). *Pearson's chi-square $P$ value $<0.05$.

$D V T=$ deep vein thrombosis; $P E=$ pulmonary embolism.

diagnosis of DVT + PE. A total of 994 (46.3\%) patients were male, and 1,009 (47.0\%) were aged 65 years or older. The average duration of the preindex and postindex period was $18.6 \pm 10.0$ (median, 17.1) and 21.3 \pm 10.0 (median, 19.2) months, respectively. Patients received a mean of $7.3 \pm 6.0$ distinct medications (of all types) during the preindex period. The history of any bleed (i.e., from the list in Table 1) in the 3 months preceding the index VTE ranged from $8.3 \%$ to $9.0 \%$, and the incidence was not significantly different among the 3 index diagnosis groups (ANOVA $\mathrm{F}=0.2 ; P=0.81$ ). Preindex medical, pharmacy, and total health care costs were similar among the 3 index diagnosis groups by ANOVA $(F<0.42$; $P>0.65$ for all comparisons).

Table 4 provides a list of risk factors stratified by index diagnosis. Patients with an active case or history of malignancy and/or cardiac diseases (excluding atrial fibrillation, congestive heart failure, and myocardial infarction) had the highest representation in the cohort.

\section{Follow-up Events and Costs}

Table 5 illustrates the number of patients during the follow-up period who had at least 1 VTE event, the number of total events observed, and the associated total cost. Overall, 534 patients $(24.9 \%$ of the original 2,147) experienced 662 VTE and bleed events requiring hospitalization during the follow-up period. A total of 288 patients experienced 361 recurrent VTE events, 169 patients experienced 211 bleed events, and 77 patients experienced 90 combined VTE + bleed events during the follow-up period. The health system incurred a weighted average of $\$ 14,975$ in extra cost per VTE event due to the occurrence of postindex events that required hospitalization. Weights were calculated based on the number of events in each event group. This translates into an additional annualized cost to the plan of $\$ 2,101$ per patient per year (PPPY) diagnosed with an incident $\operatorname{VTE}(\$ 14,975 \times[534 / 2,147$ patients $] \times[12$ months $/ 21.3$ months]). We found that 612 (28.5\%) patients suffered 1,489 bleed events that did not require hospitalization. Notably, 1,146 patients $(612+534=1,146)$ or $53.4 \%$ experienced at least 1 event in the postindex period.

Unadjusted and multivariate modeling for all pair-wise comparisons between groups yielded no significant differences in costs for all types of follow-up events requiring hospitalization. Of note, the costs to treat recurrent VTE requiring hospitalization between the PE and the DVT + PE groups did not reach statistical significance $(P=0.11)$. Although the observed difference in costs for bleeds not requiring hospitalization between the PE and DVT + PE groups was small, this difference did reach statistical significance in the multivariate model (Wald $P<0.05$ ). In all multivariate models, cardiac events, malignancy, trauma, and surgery were significantly associated with increased cost. In addition, the INR monitoring rate was not a significant term (Wald $P>0.15$ ) in any of the 4 models.

Figure 1 shows the component costs for each derived resource center by each of the 3 types of recurrent events requiring hospitalization. The percentages of total costs were consistent between groups, with room and board costs accounting for the highest percentage of total costs.

\section{Index Thrombosis Event}

The index VTE length of stay (LOS) was $5.3 \pm 4.2$ (median, 5) days for the DVT group; $8.0 \pm 5.0$ (median, 7.0 ) days for the PE group; and $8.3 \pm 3.8$ (median, 8 ) days for the DVT + PE group. Of note, $17 \%$ of patients had an LOS of $\leq 1$ day. The DVT group LOS was significantly shorter than both the PE and DVT + PE groups ( $P<0.05)$. It was observed that $211(9.8 \%)$ patients were transferred directly from the hospital to an SNF. The overall LOS for this group was $53 \pm 55$ days (median, 32), and all pair-wise comparisons between index VTE groups were not significantly different.

The overall cost for the index VTE event, including any contiguous skilled facility care, was $\$ 8,331 \pm \$ 18,667$ (median, $\$ 4,003)$. By diagnosis group, cost for the index VTE event was $\$ 7,712 \pm \$ 18,339$ (median, $\$ 3,131$ ) for DVT; $\$ 9,566 \pm$ $\$ 13,512$ (median, $\$ 6,424$ ) for PE; and $\$ 12,200 \pm \$ 24,038$ (median, \$6,678) for DVT + PE. Both the PE and DVT + PE groups had significantly higher costs compared with the DVT group $(P<0.05)$. These costs by resource center were $3.7 \%$ from the emergency department, 53.0\% from room and board, 9.7\% from both diagnostic/laboratory and pharmacy, and $23.8 \%$ from 


\section{Longitudinal Evaluation of Health Plan Cost per Venous Thromboembolism or Bleed Event}

in Patients With a Prior Venous Thromboembolism Event During Hospitalization

TABLE 5 Postindex VTE/Bleed Events and Mean Total Costs per Event by Index Diagnosis Group*

\begin{tabular}{|c|c|c|c|c|c|}
\hline \multicolumn{2}{|c|}{ Recurrent Events Requiring Hospitalization } & $\begin{array}{c}\text { DVT } \\
\mathrm{n}=1,499\end{array}$ & $\begin{array}{c}\text { PE } \\
\mathrm{n}=373\end{array}$ & $\begin{array}{c}\mathrm{DVT}+\mathrm{PE} \\
\mathrm{n}=275\end{array}$ & $\begin{array}{c}\text { Overall } \\
\mathrm{n}=2,147\end{array}$ \\
\hline VTE & $\begin{array}{l}\mathrm{N}(\%) \\
\text { No. of events } \\
\text { No. of events per patient } \\
\text { Total cost (mean } \pm \text { SD) } \\
\text { [median] }\end{array}$ & $\begin{array}{r}182(12.1) \\
221 \\
1.21 \\
\$ 11,419 \pm \$ 26,292 \\
{[\$ 4,703]}\end{array}$ & $\begin{array}{r}69(18.5) \\
90 \\
1.30 \\
\$ 11,014 \pm \$ 12,778 \\
{[\$ 7,627]}\end{array}$ & $\begin{array}{r}37(13.5) \\
50 \\
1.35 \\
\$ 19,237 \pm \$ 30,404 \\
{[\$ 7,705]}\end{array}$ & $\begin{array}{r}288(13.4) \\
361 \\
1.26 \\
\$ 12,326 \pm \$ 24,448 \\
{[\$ 5,736]}\end{array}$ \\
\hline Bleed & $\begin{array}{l}\mathrm{N}(\%) \\
\text { No. of events } \\
\text { No. of events per patient } \\
\text { Total cost }(\text { mean } \pm \text { SD) } \\
\text { [median] }\end{array}$ & $\begin{array}{r}116(7.7) \\
145 \\
1.25 \\
\$ 16,530 \pm \$ 61,634 \\
{[\$ 4,085]}\end{array}$ & $\begin{array}{r}35(9.4) \\
43 \\
1.23 \\
\$ 13,532 \pm \$ 21,470 \\
{[\$ 5,946]}\end{array}$ & $\begin{array}{r}18(6.6) \\
23 \\
1.28 \\
\$ 11,175 \pm \$ 9,851 \\
{[\$ 9,178]}\end{array}$ & $\begin{array}{r}169(7.9) \\
211 \\
1.25 \\
\$ 15,339 \pm \$ 52,029 \\
{[\$ 4,999]}\end{array}$ \\
\hline \multirow[t]{2}{*}{ VTE + bleed } & $\begin{array}{l}\mathrm{N}(\%) \\
\text { No. of events } \\
\text { No. of events per patient } \\
\text { Total cost (mean } \pm \text { SD) } \\
\text { [median] }\end{array}$ & $\begin{array}{r}44(2.9) \\
49 \\
1.11 \\
\$ 17,385 \pm \$ 35,989 \\
{[\$ 8,666]}\end{array}$ & $\begin{array}{r}23(6.2) \\
28 \\
1.22 \\
\$ 39,941 \pm \$ 108,552 \\
{[\$ 12,200]}\end{array}$ & $\begin{array}{r}10(3.6) \\
13 \\
1.30 \\
\$ 17,102 \pm \$ 14,901 \\
{[\$ 10,936]}\end{array}$ & $\begin{array}{r}77(3.6) \\
90 \\
1.17 \\
\$ 24,085 \pm \$ 65,411 \\
{[\$ 10,185]}\end{array}$ \\
\hline & Weighted average $\dagger$ & $\$ 13,920$ & $\$ 16,947$ & $\$ 16,676$ & $\$ 14,975$ \\
\hline \multicolumn{6}{|c|}{ Recurrent Events Not Requiring Hospitalization } \\
\hline Bleed & $\begin{array}{l}\mathrm{N}(\%) \\
\text { No. of events } \\
\text { No. of events per patient } \\
\text { Total cost (mean } \pm \text { SD) } \\
\text { [median] }\end{array}$ & $\begin{array}{r}413(27.6) \\
1,022 \\
2.47 \\
\$ 235 \pm \$ 367 \\
{[\$ 95]}\end{array}$ & $\begin{array}{r}115(30.8) \\
275 \\
2.39 \\
\$ 266 \pm \$ 387 \ddagger \\
{[\$ 114]}\end{array}$ & $\begin{array}{r}84(31) \\
192 \\
2.29 \\
\$ 222 \pm \$ 470 \ddagger \\
{[\$ 70]}\end{array}$ & $\begin{array}{r}612(28.5) \\
1,489 \\
2.43 \\
\$ 239 \pm \$ 386 \\
{[\$ 95]}\end{array}$ \\
\hline \multicolumn{6}{|c|}{ 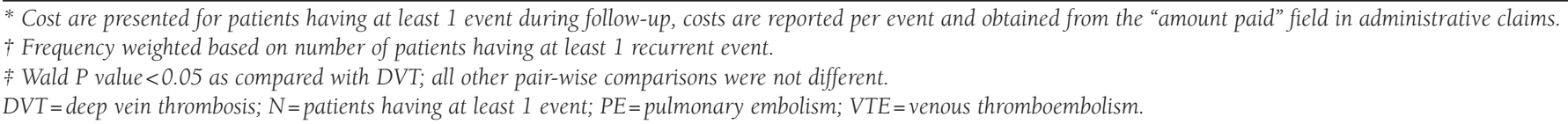 } \\
\hline
\end{tabular}

the "other" category. Significant contributors to the "other" category were operating room, anesthesia, dialysis, oxygen, and professional (medical, physical, and occupational therapy) fees.

\section{Anticoagulation Therapy and Monitoring Secondary Outcomes}

A total of 2,090 patients received warfarin therapy after the index VTE event, 283 (13.2\% of 2,147 study patients) in combination with LMWH. Average therapy time for the DVT group was $6.2 \pm 6.0$ (median, 4.4) months; for the PE group, $7.9 \pm 6.0$ (median 6.3) months; and for the DVT + PE group, $8.0 \pm 7.0$ (median, 6.2) months. Duration of therapy was significantly longer in both the PE and the DVT + PE groups compared with the DVT group $(P<0.05)$. Overall, the average warfarin cost was $\$ 130 \pm \$ 174$ (median, $\$ 72$ ), or $\$ 19.40$ per utilizing patient per month (PPPM). Of note, 361 patients received an outpatient course of LMWH therapy, with a duration and cost of $14.2 \pm 23.0$ days (median, 7 ); and $\$ 703 \pm \$ 1,540$ (median, $\$ 316 ; \$ 50$ per patient-day), respectively. No difference was observed between groups with respect to the duration or cost of LMWH therapy.

For patients receiving warfarin, a patient received a mean of $13.5 \pm 13.0$ (median, 10) PT/INR tests or 1.86 tests PPPM over the course of warfarin therapy. PT/INR test rates were nearly identical for all index diagnosis groups. The average cost for PT/INR testing was $\$ 84 \pm \$ 220$ (median, $\$ 42$ ), or $\$ 12.50$ PPPM. Additionally, 611 (29.2\%) patients had at least 1 office visit within a day of the PT/INR laboratory performance; the cost for these office visits potentially attributable to warfarin monitoring was $\$ 127 \pm 162$ (median, \$79), or \$18.96 PPPM.

\section{Discussion}

In this analysis, we conducted a retrospective cohort study of patients gathered from 2 large U.S. managed care populations with the primary objective of determining the cost and utilization of medical resources associated with managing VTE follow-up events. This population was identified by the occurrence of an index VTE hospital event and was at high risk for VTE (e.g., 59\% overall with history of malignancy or active malignancy), allowing observation of customary management of VTE outside of a controlled clinical trial. Of the 2,147 patients identified with an incident VTE hospital event, 534 patients (24.9\%) experienced at least 1 event requiring hospitalization over an average follow-up time of 21.3 (median, 19.2) months. In addition, a total of 612 patients (28.5\%) experienced at least 1 bleed event during the follow-up period that did not require hospitalization 


\section{Longitudinal Evaluation of Health Plan Cost per Venous Thromboembolism or Bleed Event}

in Patients With a Prior Venous Thromboembolism Event During Hospitalization

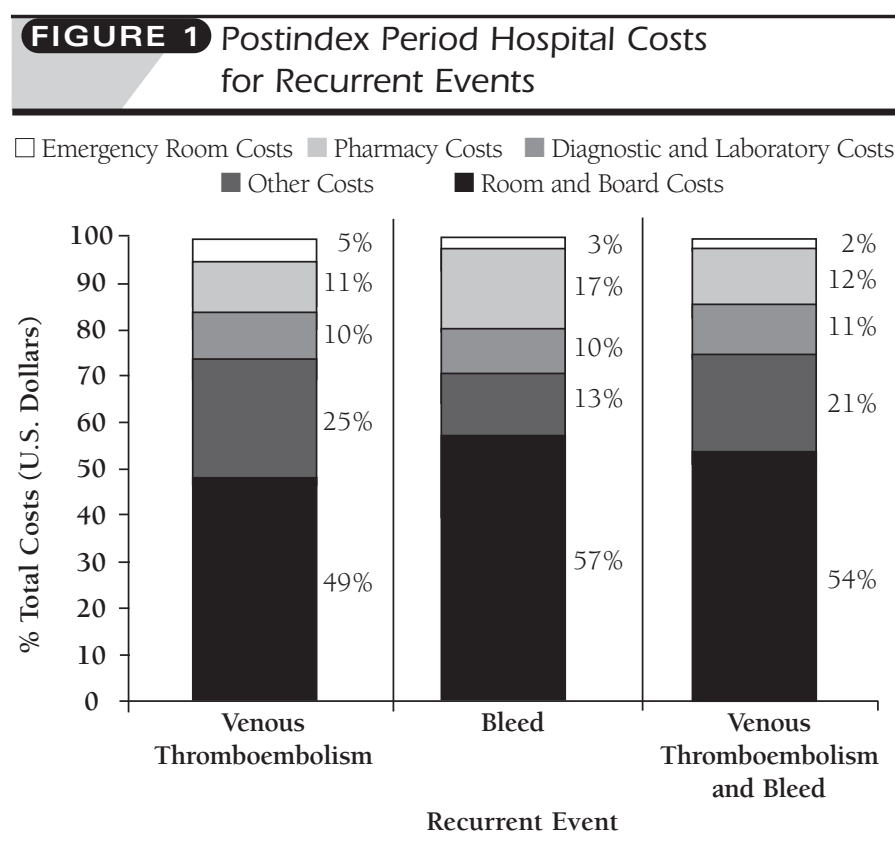

Hospital claims that were paid based upon prospective (e.g., per-diem) rates were adjusted to a calculated paid-to-charge ratio for each resource center. Emergency room included all emergent and urgent care services universal billing (UB, [UB-92 range: 450-459]); room and board included all costs related to critical care units, wards, nursing, monitoring, and supply costs (UB-92 range: 100-249, 279-299, 620-624, 700-770); diagnostic and laboratory included all laboratory and diagnostic tests, such as radiographic tests, Doppler ultrasound, computed tomography, resonance-based diagnostic tests, and peripheral vascular laboratory costs (UB-92 range: 300-359, 402-409, 610-619, 921); and pharmacy included all medication costs and intravenous supplies and administration (UB-92 range: 250-269). All paid amounts that contained UB-92 codes that were not part of the aforementioned ranges were allocated to other costs; significant contributors in this category were operating room, anesthesia, dialysis, oxygen, and professional (medical, physical, and occupational therapy) fees.

(i.e., outpatient bleed). The "average" inpatient recurrent VTE/bleed event cost the health plan $\$ 14,975$, or $\$ 2,101$ PPPY, for recurrent VTE/bleed events in patients with an initial VTE diagnosis. In addition, our findings show that room and board contributed more than half the costs of the VTE follow-up management, indicating management strategies that shorten LOS are likely to be important in reducing cost.

The costs revealed by our research may help MCO decision makers evaluate the budgetary impact of disease management programs and new interventions. While anticoagulation pharmacy costs are a relatively small $(<1 \%)$ component of overall costs, improved pharmacy management has the potential to positively impact costs from other resource centers by reducing LOS or need for other services. Since our study quantified costs on a per-event basis, various decision analyses can be employed at the local health plan level to estimate potential cost savings between interventions with differing costs as well as their associated event rates.
Previous observational studies that were either economically based or formed a component of a clinical trial directly compared the savings or cost-effectiveness of LMWH with UFH. These studies focused on a wide variety of settings and management strategies, such as prospective outpatient management of recurrent thromboembolism events (100, 125, and 107 patients) ${ }^{29-31}$; randomized, multicenter trials $^{16,32,33}$; decision modeling approaches ${ }^{16,18}$; and home-oriented pharmacy management. ${ }^{34}$ Two observational studies focused on detailing DVT costs of care from the MCO perspective over the first 90 days: (1) a prospective study inside a group-model $\mathrm{HMO}^{35}$ and (2) a retrospective study conducted in a staff-model HMO. ${ }^{36}$

Spyropoulos et al., in a retrospective staff-model $\mathrm{HMO}$ study, followed 129 patients with DVT and reported an average cost of care for the first 90 days of between $\$ 9,347$ and $\$ 11,930$, depending on whether the initial treatment consisted of LMWH or UFH. The event rate over the follow-up period was found to be similar among groups, although much lower than in our analysis. The average initial hospitalization cost was between $\$ 4,849$ and $\$ 8,501$, a finding similar to our study. ${ }^{36}$ Tillman et al. followed 391 patients with DVT in a group-model $\mathrm{HMO}$, all acutely treated with $\mathrm{LMWH}$, and reported an estimated cost of $\$ 4,695$ per initial hospitalization. ${ }^{35}$

One retrospective observational study conducted in a realworld setting detailed the cost of VTE care by using discharge data from 6 states. $^{15}$ The study had a payer mix of $56.0 \%$ Medicare, $22.5 \%$ managed care, and $21.5 \%$ not reported. The average cost of initial hospitalization was $\$ 5,779, \$ 9,476$, and $\$ 11,189$ for DVT, DVT + PE, and DVT with major bleed, respectively. Report on follow-up, from only 1 of the original 6 states, noted a 9.5\% 6-month DVT recurrence rate with an average cost of $\$ 6,946$.

Our study built on this previous research by including a larger sample size, a longer duration of follow-up, actual costs for all types of resources, and data from 2 geographically disparate MCOs. We also placed more emphasis on detailing and comparing cost drivers and costs to treat recurrent events on a per-event basis.

In addition, our data complement Anderson's recent cost analysis of pharmacist-driven, outpatient anticoagulation services for atrial fibrillation patients on chronic warfarin therapy. ${ }^{37}$ First, he found that anticoagulation medication costs were \$19.09 PPPM, nearly identical to the $\$ 19.40$ PPPM result reported in our study. Second, our data provide the means to perform the cost-avoidance analysis that Anderson suggested be performed to determine the true cost impact of an outpatient oral anticoagulant monitoring service since we provide the cost of events that occur despite anticoagulant therapy. Future research might include a cost-avoidance/impact analysis comparing current anticoagulant strategies with newer therapies with different acquisition costs and effectiveness/risk parameters. 


\section{Longitudinal Evaluation of Health Plan Cost per Venous Thromboembolism or Bleed Event in Patients With a Prior Venous Thromboembolism Event During Hospitalization}

\section{Limitations}

Several limitations to the present study should be noted. First, due to the retrospective design of our study, only associations can be calculated between the variables of interest and the outcomes (i.e., it is not possible to speculate about causality).

Second, although multivariate techniques were used, administrative claims data do not account for all potential confounders. Although these techniques are well accepted in determining adjusted estimates, they cannot adjust for all differences present in the case mix because certain variables (e.g., race, socioeconomic status, hospital type) are not available.

Third, coding errors have been well documented in administrative claims data. Up-coding, the existence of a VTE or bleed code as part of a rule-out admission, may have existed during the follow-up period in our study. Additionally, patients with a VTE prior to the preindex period could be included in this study, which would tend to select higher-risk patients compared with a longer preindex period (i.e., a patient with a history of VTE events would be more likely to be admitted to our study). Other studies attempting to measure such events have observed a recurrent VTE rate of $5.1 \%$ to $8.6 \%,{ }^{6,738}$ and a bleed rate requiring admission of $2.6 \%$ and $2.7 \% .{ }^{6.7}$ However, none of these studies observed patients outside the constructs of a clinical trial, highly specialized clinic, or other tertiary centers of care; thus, it is plausible for our rates of recurrent VTE and bleed to be higher. In addition, although this rate of up-coding is not known, this postulated vigilance toward rule-out admissions does represent a cost to the system and should be factored into the mean costs. Also, the effect of including up-coding in the analysis would be expected to decrease the mean cost per event.

Fourth, there were 77 patients with a recurrent VTE and bleed event requiring hospitalization. It would be prudent to assume that a proportion of these events represented a bleed event alone rather than a recurrent VTE and a subsequent inpatient bleed. However, it should be noted that the average cost per VTE and bleed was approximately 57\% and 97\% higher than just a bleed or VTE, respectively. This trend in mean costs suggests that a higher volume of care may have been provided to patients with VTE and bleed. Future research should be undertaken to combine hospital chart level data with administrative claims data to assist in verifying VTE diagnoses and provide greater depth and granularity of data from the inpatient setting.

Fifth, we only studied the costs associated with adverse events and INR monitoring. Potential new therapies may be associated with other adverse outcomes or may require laboratory monitoring other than INR.

Sixth, this evaluation did not include an examination of the effect of pharmacy benefit design and coverage on clinical or economic outcome. Nearly one half (47\%) of the study population was older than 65 years. We did not measure the proportion of this population who were members of a Medicare $\mathrm{HMO}$ with a pharmacy benefit that included biannual or annual benefit dollar maximums; the drug benefit annual maximums varied based on the county of residence. Out-of-pocket cost may have been a factor in adherence to drug therapy including warfarin, although the average wholesale cost of warfarin was only about $\$ 30$ per month of therapy at the time of this study. Nevertheless, additional analysis of these patients, their specific pharmacy benefit design, and their adherence to warfarin therapy may provide insight regarding the impact of benefit design on health outcomes in this age group.

Seventh, administrative claims capture neither mortality nor the clinical data necessary to permit classification of major and minor bleeds. Hence, we used hospitalized/not hospitalized as a proxy for bleed severity to describe the events since these were measures we could retrieve from an administrative claims database. Due to the absence in medical claims of specific J-codes available at the time of this study, LMWH was captured in the outpatient setting only from pharmacy claims, which would tend to leave the true cost of LMWH therapy in this patient population underreported.

Eighth, we did not have the ability to assess the effects of an anticoagulation management clinic on the incidence of rehospitalization and associated expenses. The presence of an anticoagulation clinic would be expected to decrease the adverse events that precipitated repeat hospitalizations.

Finally, a total of 100 patients (4.7\%) had ICD-9 codes that could have been for heparin-induced thrombocytopenia (HIT); 67 patients (3.1\%), unspecified thrombocytopenia (codes 287.5); 21 patients $(0.98 \%)$, secondary thrombocytopenia (drug-induced, code 287.4); and 6 patients (28.0\%) had codes for both. These results are in agreement with estimates for the prevalence of HIT from $0.2 \%$ to $5 \% .{ }^{39,40}$ Costs for HIT patients were not specifically captured and are included in the total costs.

\section{Conclusions}

We observed costs attributable to VTE care in a longitudinal analysis from a managed care payer perspective. We focused on the cost per event so that managed care decision makers can better evaluate local health plan formularies for existing and new medication therapies and programs such as anticoagulation clinics. Since bleed and recurrent VTE events that require rehospitalization may occur in approximately $25 \%$ of patients presenting with an initial VTE event and cost the health plan approximately $\$ 15,000$ per event, management strategies and therapeutic options are worthwhile considerations for MCOs.

\section{ACKNOWLEDGMENT}

The authors wish to thank J. Matthew Groesbeck, BS, medical writer, HealthCore, Inc., Wilmington, DE, for his assistance with this research 


\section{Longitudinal Evaluation of Health Plan Cost per Venous Thromboembolism or Bleed Event in Patients With a Prior Venous Thromboembolism Event During Hospitalization}

\section{DISCLOSURES}

Funding for this research was provided by AstraZeneca LP, the manufacturer of ximelagatran, and was obtained by authors Vincent Willey and Ole Hauch; Hauch and author Gail Wygant are employed by AstraZeneca LP. Willey and author Michael F. Bullano disclose that they have received research grants as paid consultants to pharmaceutical companies; author Alex C. Spyropoulos discloses that he is on the speaker's bureau and is a consultant to Aventis and is a consultant to AstraZeneca LP and Sanofi-Synthelabo; Lauren Hoffman discloses no potential bias or conflict of interest relating to this article.

Bullano served as principal author of the study. Study concept and design were contributed by Bullano, Willey, Hauch, and Wygant. Analysis and interpretation of data were contributed by all authors. Drafting of the manuscript was primarily the work of Bullano and Willey, and its critical revision was the work all authors. Statistical expertise was contributed by Bullano, and administrative, technical, and/or material support was provided by Hoffman.

\section{REFERENCES}

1. Anderson FA Jr., Wheeler HB, Goldberg RJ, et al. A population-based perspective of the hospital incidence and case-fatality rates of deep vein thrombosis and pulmonary embolism. The Worcester DVT Study. Arch Intern Med. 1991;151:933-38.

2. Goldhaber SZ. Thrombolysis for pulmonary embolism. Prog Cardiovasc Dis. 1991;34:113-34

3. Salzman EW, Hirsh J. The epidemiology, pathogenesis, and natural history of venous thrombosis. In: Colman RW, Hirsh J, Marder VJ, Salzman EW, eds. Hemostasis and Thrombosis: Basic Principles and Clinical Practice. 3rd ed. Philadelphia, PA: JB Lippincott Company; 1994:1275-96.

4. Mamdani MM, Racine E, McCreadie S, et al. Clinical and economic effectiveness of an inpatient anticoagulation service. Pharmacotherapy. 1999; 19:1064-74.

5. Estrada CA, Mansfield CJ, Heudebert GR. Cost-effectiveness of low-molecularweight heparin in the treatment of proximal deep vein thrombosis. J Gen Intern Med. 2000;15:108-15.

6. Prandoni P, Lensing AW, Cogo A, et al. The long-term clinical course of acute deep venous thrombosis. Ann Intern Med. 1996;125:1-7.

7. The Columbus Investigators. Low-molecular-weight heparin in the treatment of patients with venous thromboembolism. N Engl J Med. 1997;337:657-62.

8. Bick RL. Proficient and cost-effective approaches for the prevention and treatment of venous thrombosis and thromboembolism. Drugs. 2000;60:575-95.

9. Spyropoulos AC. Outpatient-based treatment protocols in the management of venous thromboembolic disease. Am J Manag Care. 2000;6:S1034-S1044.

10. Anonymous. Proceedings of the American College of Chest Physicians 5th Consensus on Antithrombotic Therapy. Chest. 1998;114:439S-769S.

11. Hirsh J, Dalen J, Guyatt G. The sixth (2000) ACCP guidelines for antithrombotic therapy for prevention and treatment of thrombosis. American College of Chest Physicians. Chest. 2001;119:1S-2S

12. White RH, Zhou H, Romano PS. Length of hospital stay for treatment of deep venous thrombosis and the incidence of recurrent thromboembolism. Arch Intern Med. 1998;158:1005-10.

13. Racine E. Justifying high-cost anticoagulant therapy. Am J Health Syst Pharm. 2002;59:S18-S20.

14. Bick RL. Therapy for venous thrombosis: guidelines for a competent and cost-effective approach. Clin Appl Thromb Hemost. 1999;5:2-9.

15. O'Brien JA, Caro JJ. Direct medical cost of managing deep vein thrombosis according to the occurrence of complications. Pharmacoeconomics. 2002;20: 603-15.

16. de Lissovoy G, Yusen RD, Spiro TE, et al. Cost for inpatient care of venous thrombosis: a trial of enoxaparin vs standard heparin. Arch Intern Med. 2000; 160:3160-65
17. Gould MK, Dembitzer AD, Sanders GD, Garber AM. Low-molecularweight heparins compared with unfractionated heparin for treatment of acute deep venous thrombosis. A cost-effectiveness analysis. Ann Intern Med. 1999; 130:789-99.

18. Rodger M, Bredeson C, Wells PS, et al. Cost-effectiveness of low-molecularweight heparin and unfractionated heparin in treatment of deep vein thrombosis. CMAJ. 1998;159:931-38

19. van den Belt AG, Bossuyt PM, Prins MH, Gallus AS, Buller HR. Replacing inpatient care by outpatient care in the treatment of deep venous thrombosisan economic evaluation. TASMAN Study Group. Thromb Haemost. 1998;79: 259-63.

20. Medi-Span WKH. Master Drug Database (MDDB) Generic Product Identifier; 2003

21. Schneeweiss S, Seeger JD, Maclure M, et al. Performance of comorbidity scores to control for confounding in epidemiologic studies using claims data. Am J Epidemiol. 2001;154:854-64.

22. Hyers TM, Agnelli G, Hull RD, et al. Antithrombotic therapy for venous thromboembolic disease. Chest. 2001;119:176S-193S.

23. Go AS, Hylek EM, Chang Y, et al. Anticoagulation therapy for stroke prevention in atrial fibrillation: how well do randomized trials translate into clinical practice? JAMA. 2003;290:2685-92

24. Rosner B. Fundamentals of Biostatistics. 4th ed. Belmont, CA: Wadsworth; 1995:306-19.

25. Gleason J. Improved confidence intervals for binomial proportions. Stata Technical Bulletin. 1999:9:208-11.

26. Diehr P, Yanez D, Ash A, Hornbrook M, Lin DY. Methods for analyzing health care utilization and costs. Annu Rev Public Health. 1999;20:125-44.

27. Raftery AE. Bayesian model selection in social research. In: Marsden PV, ed. Sociological Methodology. Cambridge, MA: Blackwells; 1995:111-96.

28. STATA Corporation LP. College Station, TX; 2004

29. Groce JB, III. Patient outcomes and cost analysis associated with an outpatient deep venous thrombosis treatment program. Pharmacotherapy 1998;18:175S-180S

30. Pearson SD, Blair R, Halpert A, Eddy E, McKean S. An outpatient program to treat deep venous thrombosis with low-molecular-weight heparin. Eff Clin Pract. 1999:2:210-17

31. Ting SB, Ziegenbein RW, Gan TE, et al. Dalteparin for deep venous thrombosis: a hospital-in-the-home program. Med J Aust. 1998;168:272-76.

32. Boccalon H, Elias A, Chale JJ, Cadene A, Gabriel S. Clinical outcome and cost of hospital vs home treatment of proximal deep vein thrombosis with a low-molecular-weight heparin: the Vascular Midi-Pyrenees study. Arch Intern Med. 2000;160:1769-73.

33. Hull RD, Raskob GE, Rosenbloom D, et al. Treatment of proximal vein thrombosis with subcutaneous low-molecular-weight heparin vs intravenous heparin. An economic perspective. Arch Intern Med. 1997;157:289-94.

34. Dedden P, Chang B, Nagel D. Pharmacy-managed program for home treatment of deep vein thrombosis with enoxaparin. Am J Health Syst Pharm. 1997:54:1968-72

35. Tillman DJ, Charland SL, Witt DM. Effectiveness and economic impact associated with a program for outpatient management of acute deep vein thrombosis in a group model health maintenance organization. Arch Intern Med. 2000;160:2926-32.

36. Spyropoulos AC, Hurley JS, Ciesla GN, de Lissovoy G. Management of acute proximal deep vein thrombosis: pharmacoeconomic evaluation of outpatient treatment with enoxaparin vs inpatient treatment with unfractionated heparin. Chest. 2002;122:108-14.

37. Anderson RJ. Cost analysis of a managed care decentralized outpatient pharmacy anticoagulation service. J Manag Care Pharm. 2004;10(2):159-65. 


\section{Longitudinal Evaluation of Health Plan Cost per Venous Thromboembolism or Bleed Event}

in Patients With a Prior Venous Thromboembolism Event During Hospitalization

38. Brandjes DP, Heijboer H, Buller HR, et al. Acenocoumarol and heparin compared with acenocoumarol alone in the initial treatment of proximal-vein thrombosis. N Engl J Med. 1992;327:1485-89.

39. Andreescu AC, Possidente C, Hsieh M, Cushman M. Evaluation of a pharmacy-based surveillance program for heparin-induced thrombocytopenia. Pharmacotherapy. 2000;20:974-80.

40. Lee D, Carter C, Dolan S. Practical treatment guidelines, heparin-induced thrombocytopenia. 2004. Available at: www.tigc.org/pdf/hit04.pdf. Acccessed June 15, 2005. 